\title{
O PAPEL DO FARMACÊUTICO COMUNITÁRIO NO RASTREAMENTO DO DIABETES MELLITUS TIPO II
}

\author{
THE ROLE COMMUNITY PHARMACIST IN SCREENING FOR TYPE II \\ DIABETES MELLITUS
}

\section{Fabio Junio da Silva Melo ${ }^{1}$}

RESUMO: Objetivo: Promover um estudo amplo, coerente e participativo sobre o papel do Farmacêutico no rastreamento do Diabetes Mellitus Tipo II, realizado nas farmácias comunitárias, como instrumento importante na atenção Primária a saúde da População. Método: consiste numa revisão de estudos publicados no período entre janeiro de 2013 a janeiro de 202I em Revistas Brasileiras de Ciências Farmacêuticas SaBios, Scielo, Revista Ibero-americana de humanidades, ciências e educação, que abrangeram com relevância a Diabetes Mellitus tipo II. Estes artigos foram publicados na base de Dados Google Acadêmico. Resultados: de um total de 28 trabalhos científicos, foram encontrados 7 artigos relacionados diretamente ao cuidado farmacêutico ao paciente diabético, mas nenhum deles descreve a importância da aplicabilidade do rastreio da diabetes feito por farmacêuticos na farmácia comunitária. Conclusão: Faz- se necessário, desenvolver trabalhos de formação continuada para capacitar os farmacêuticos que atuam nas farmácias comunitárias, principalmente no que se refere as doenças crônicas não transmissíveis, especificamente na atenção voltada para o rastreamento do diabetes mellitus tipo II.

Palavras-chaves: Doenças crônicas não transmissíveis. Diabetes mellitus. Farmácia comunitária. Serviços farmacêuticos.

ABSTRACT: Objective: Promote a coherent and participatory broad study on the role of the pharmacist in the screening of type 2 diabetes mellitus, carried out in community pharmacies as an important instrument in primary health care for the population. Method: consists of a review of studies published between January 2013 and January 2021 in the Brazilian Journal of pharmaceutical sciences, SaBios, sciELO, Ibero-american journal of humanities sciences and education that covered with relevant type 2 diabetes mellitus. These articles were published in the Google academic database. Results: Of a total of 28 scientific papers, 7 articles were found directly related to pharmaceutical care for diabetic patients, but none of them describes the importance of the applicability of diabetes screening performed by a pharmacist in the community pharmacy.Conclusion: Phase, if necessary, to develop ongoing trading works to train pharmacists who work in community pharmacies, especially with regard to chronic non-communicable diseases, specifically in the attention focused on the screening of type 2 diabetes mellitus.

Keywords: Chronic non-communicable diseases. Diabetes mellitus. Community pharmacy. Pharmaceutical services.

\footnotetext{
${ }^{\mathrm{I}}$ Especialista em Farmácia Hospitalar - 202I Especialista em Farmácia clínica com ênfase em serviços farmacêuticos - 2020 Bacharel em Farmácia pela Universidade Nove de Julho (2018). Atualmente é farmacêutico Responsável Técnico na Raia Drogasil. E-mail: fabio_junior87@hotmail.com.
} 


\section{INTRODUÇÃO}

Entende-se por farmácia comunitária, a unidade técnico-administrativa de fácil acesso a população e que comercializa produtos farmacêuticos e correlatos, além de prestar serviços farmacêuticos altamente especializados, por profissional legalmente habilitado.

De acordo com a lei 13.021/2014, Farmácia é uma unidade de prestação de serviços destinados a prestar assistência farmacêutica, assistência à saúde e orientação sanitária individual e coletiva, na qual se processe a manipulação e /ou dispensação de medicamentos magistrais, oficinais, farmacopeicos ou industrializados, cosméticos, insumos farmacêuticos, produtos farmacêuticos e correlatos $^{1}$.

Faz parte do grupo das doenças crônicas não transmissíveis, o câncer, diabetes, doenças respiratórias crônicas, hipertensão, doenças do aparelho circulatório, constituindo a maior carga de morbimortalidade no mundo. Essas patologias acometem pessoas de todas as camadas sociais, sendo mais prevalecentes em populações de baixa escolaridade e renda devido às dificuldades encontradas de continuidade do tratamento $e$ acesso as informações de qualidades sobre $o$ monitoramento e acompanhamento dessas patologias.

As mortes por Doenças Crônicas não Transmissíveis acarretam perda de qualidade de vida, limitações e incapacidades, sendo responsáveis por $63 \%$ das mortes globais e afetam predominantemente os países em desenvolvimento, nos quais cerca de um terço dos óbitos ocorrem em pessoas com menos de 60 anos de idade, enquanto nos países desenvolvidos a mortalidade prematura (faixa etária de 30 a 69 anos) corresponde a menos de $13 \%$ dos $\operatorname{casos}^{2}$.

O farmacêutico que atua nas farmácias comunitárias tem a grande oportunidade de colaborar com o sistema de saúde por meio dos serviços farmacêuticos prestados à população. Os serviços farmacêuticos variam desde a educação em saúde até aqueles mais especializados como aplicação de injetáveis, consulta farmacêutica, acompanhamento da farmacoterapia do paciente, serviços de rastreabilidade de algumas doenças como, diabetes, hipertensão, distúrbios do 
colesterol entre outras. De acordo com as diretrizes de atenção primária a saúde é possível identificar as causas, os fatores de riscos, estratificar do grau de avanço da doença como estratégia de diminuição de mortes prevalecente no Brasil resultante da doença não tratada de forma correta.

Nos últimos 30 anos vem ocorrendo um forte movimento no Brasil em busca de ampliação da atuação do farmacêutico e da participação da farmácia comunitária no sistema de saúde brasileiro. Esse processo pode ser evidenciado pela portaria nº 3.916 de 1998 do Ministério da Saúde (MS) que estabeleceu a "Política nacional de medicamentos" (PNM), sendo uma das diretrizes a reorientação da assistência farmacêutica e da resolução no 338 de 2004 do Conselho Nacional de Saúde (CNS) que estabeleceu a Política Nacional de Assistência Farmacêutica ${ }^{3}$.

O farmacêutico é tradicionalmente reconhecido como um profissional responsável pela produção e distribuição de medicamentos e pouco envolvido no cuidado dos pacientes. O modelo de pratica de dispensação de medicamentos também corresponde a esse papel tradicional. Essa realidade, entretanto, vem sendo transformada, na medida em que cresce a necessidade de uma participação do farmacêutico no manejo do uso dos medicamentos, tendo como foco de trabalho o paciente e não apenas o medicamento ${ }^{4}$.

O Diabetes Mellitus é uma doença caracterizada pelo aumento da glicose no sangue decorrente de defeitos na secreção ou ação da insulina no organismo. A insulina é produzida pelas cédulas beta pancreáticas tem como papel fundamental promover a entrada de glicose nas células para que estas possam obter energia e realizar suas funções. O Diabetes Mellitus pode ser dividido em Tipo I e Tipo II, objeto de estudo deste trabalho5.

Segundo a Organização Mundial da saúde a DM é considerada como uma epidemia mundial e um problema de saúde pública, com crescente prevalência e considerada em todo mundo. Até 2025, a expectativa é de 250 milhões de pessoas acometidas pela doença e no Brasil serão 18,5 milhões ${ }^{6}$.

O rastreamento do diabetes tipo II pode ser desenvolvido por meio de uma consulta farmacêutica em ambiente confortável e privado da farmácia para realizar a 
avaliação do perfil glicêmico do paciente, conhecer a história da doença e orientar sobre as complicações e comorbidades. Os pacientes recebem ao final do atendimento um relatório detalhado dos resultados obtidos e que podem ser compartilhados com médicos e demais profissionais da saúde sem finalidade de diagnóstico que servirá como parâmetro para futuro diagnóstico médico, acompanhamento da farmacoterapia e controle da doença promovendo dessa forma a melhoria da a qualidade de vida dos pacientes e familiares.

\section{METODOLOGIA}

A metodologia desenvolvida neste trabalho consiste numa revisão de estudos publicados no período entre janeiro de 2013 a janeiro de 2021 em Revistas Brasileiras de Ciências Farmacêuticas SaBios, Scielo, Revista Ibero-americana de humanidades, ciências e educação, que abrangeram com relevância a Diabetes Mellitus tipo II.

Estes artigos foram publicados na base de Dados Google Acadêmico. Após a pesquisa foram analisados criteriosamente os estudos mais relevantes referentes ao tema citado de forma a promover uma educação continuada e assertiva para os farmacêuticos que atuam no seguimento de farmácias comunitárias tendo em vista contribuir para a melhoria da qualidade de vida da população pré-diabética subdiagnosticada bem como colaborar para o uso racional do medicamento.

\section{REVISÃO DA LITERATURA}

Com o aumento da expectativa de vida da população brasileira, dados do ministério da saúde apontam que em 2050 podemos ocupar a quinta maior população idosa do mundo, chegando aproximadamente a 2 bilhões de pessoas com mais de 60 $\operatorname{anos}^{7}$.

Em 2015, a Federação Internacional de diabetes estimou que $8,8 \%$ da população mundial com 20 a 79 anos (415 milhões de pessoas) viviam com diabetes. Com a persistência dessa tendência, o número de pessoas pode ser superior a 642 milhões em 2040. O Brasil estava em $4^{\underline{O}}$ lugar na lista dos io países com maior 
número de indivíduos com esta patologia no ano de 2015 (14,3 milhões), sendo que as projeções para o ano de 2040 demonstram 23,3 milhões de pessoas ${ }^{8}$.

Um estudo publicado na Research, Society and Development, v. Io, n. I4, 2021, demonstrou que a aplicação da atenção farmacêutica se utilizando como ferramenta, a consulta farmacoterapêutica, permite identificar e propor soluções de problemas que muitas vezes não relatado ao médico ou que esse não tenha percebido 9 .

Dezenas de ensaios clínicos demonstram que a intervenção farmacêutica produz reduções adicionais na Aic entre 0,5\% e I,0\% em comparação ao cuidado usual recebido pelos pacientes ${ }^{\mathrm{IO}}$.

Um estudo de metanalise randomizado avaliou o impacto das intervenções providenciadas pelo cuidado farmacêutico no diabetes mellitus tipo 2 envolvendo 2.325 participantes demonstrou heterogeneidade elevada ( $\mathrm{I}^{2}>97 \%$; $\mathrm{p}<\mathrm{O}, \mathrm{ooI}$ ), redução nos níveis de hemoglobina glicada (-I,07\%; IC95\%: -I,32; -0,83; p<0,ooI), glicose (-29,9I $\mathrm{mg} / \mathrm{dl} ; \mathrm{IC} 95 \%$ : -43,2; -16,6; $\mathrm{p}<0.00 \mathrm{I})^{\mathrm{II}}$.

Ferreira et. Al., avaliou as dificuldades na realização do tratamento de usuários de insulina, e observou um aumento na adoção das canetas aplicadoras e diminuição do uso das seringas, que geram dificuldades em seu manuseio, e consequentemente falhas no controle glicêmico, apontando a importância do Farmacêutico na orientação do paciente e melhorando a adesão ao tratamento ${ }^{12}$.

Um estudo publicado na Revista Saúde Santa Maria edição 47 em 202I avaliou os despachos clínicos de um paciente com diabetes tipo 2 em uso de insulina, assistido pelo cuidado farmacêutico realizado por residentes em saúde da família em UBS do município de Piraquara, PR e após diversas intervenções farmacêuticas ao longo de 6 meses de acompanhamento farmacoterapêutico, obteve uma redução de $2,06 \%$ na hemoglobina glicada e de $206 \mathrm{mg} / \mathrm{ml}$ na glicemia de jejum ${ }^{13}$.

\section{CONSIDERAÇÕES FINAIS}

Mediante estudos publicados, observa-se a importância do cuidado farmacêutico voltado para o paciente diabético, bem como sua contribuição para o rastreamento dessa doença em farmácias comunitárias, considerando que o público 
alvo frequenta a farmácia mensalmente para adquirir medicamentos, produtos para a saúde e correlatos. Com o rastreamento do diabetes realizados na farmácia comunitária melhora a atenção primaria a saúde e qualidade de vida da população.

\section{CONCLUSÃO}

Faz- se necessário, desenvolver trabalhos de formação continuada para capacitar os farmacêuticos que atuam nas farmácias comunitárias, principalmente no que se refere as doenças crônicas não transmissíveis, especificamente na atenção voltada para o rastreamento do diabetes mellitus tipo II.

\section{REFERÊNCIA BIBLIOGRÁFICA}

I. Manual de orientação ao Farmacêutico e a valorização do profissional, CRF-SP, Secretaria dos colaboradores grupo Farmacia estabelecimento de saúde, sao Paulo, 2015.

2. MALTA, D.C; ANDRADE, S.S.C. A; OLIVEIRA, T. P. MOURA, L. PRADO, R, R; SOUZA, M.F.M. - Probabilidade de Morte prematura por Doenças Cronicas Nao-Transmissíveis, Brasil e regiões, projeções para 2025, Rev.Bras.Epidemiol 2019.

3. SARMENTO, D.P. AUGUSTO, C.A.M.F. CARBONI, C.P. MELLO, D.R. O farmacêutico Clinico na Farmácia Comunitaria, Rev. Eletronica gestão e Saude,vol.r-2020

4. CORRER, C.J; OTUKI, M.F. - A Prática Farmacêutica na Farmácia Comunitária, - Porto Alegre, Artmed, 2013.

5. https://www.infoescola.com - acessado em 29/06/2021 as irh e $17 \mathrm{~min}$.

6. LIMA, R; FUNGHETTO, S.S; VOLPE, W.S; FUNEZ, M.I; STIVAL, M.M. Qualidade de vida e o tempo do diagnóstico do diabetes mellitus em idosos, Rev. Bras. Gerontologia, RJ 2018.

7. www.ministériodasaúde.gov.br - acessado em o6 de fevereiro de 2022.

8. https://www.sbd.com.br - acessado em 30/or/2021 as Ioh e $35 \mathrm{~min}$.

9. GROTA,A.J.A;SILVA,D.C;FIGUEIREDO,G.A.T.O;CASTRO,R.S. Consulta e diagnóstico farmacêutico da Diabetes Mellitus tipo 2: uma revisão sistemática, Research, Society and Development, v. Io, n. I4,202I. 
ı. CORRER, C.J; REIS, W.C. T; Manual 3: diabetes em dia, I⿳亠丷厂 edição atualizada.Ed. Practice, Curitiba,2or6.

II. NOGUEIRA,M;OTUYAMA,L.J;ROCHA,P,A;PINTO.V.B,intervençoes farmaceuticas no diabetes mellitus tipo 2: uma metanalise de enssaios clinicos randomizados Hospital das Clínicas, Faculdade de Medicina, Universidade de São Paulo, São Paulo, SP, Brasil,202o.

12. SOUZA,VICTORH.M.P;MONCAO,ANNEC.M;VALLADARES,HEITOR O;MELLO,SAVIO D.P;PEDROZA,GIULIA G.O;FERREIRA, MARCELA S.; Diabetes Mellitus: Dificuldades na realização do tratamento de usuarios de insulina. Revista saúde Sta Maria,2021.

13. BAYER,MILLENA; BORBA,HELENA H.L. Impacto do cuidado farmecutico nos desfechos clinicos de um paciente com diabetes mellitus tipo 2 em uso de insulina:relato de caso. Revista Saúde Sta Maria, 202I. 